\title{
Fatiga laboral y ausentismo en el trabajo en mujeres auxiliares de enfermería de un hospital de alta complejidad, Chile
}

\author{
Occupational fatigue and work absenteeism in female assistant \\ nurses of a high-complexity hospital, Chile
}

Carolina Luengo Martínez (https://orcid.org/0000-0002-6541-3645) ${ }^{1}$

Sebastián Palma Moraga (https://orcid.org/0000-0001-8727-8141) ${ }^{1}$

Carla Sandoval Paredes (https://orcid.org/0000-0002-2481-0572) ${ }^{1}$

Amanda Sepúlveda Vásquez (https://orcid.org/0000-0003-0049-4541) ${ }^{1}$

Cristina Maccarena Villarroel Villarroel (https://orcid.org/0000-0001-6752-9062) ${ }^{1}$

\footnotetext{
${ }^{1}$ Departamento Enfermería, Universidad del Bio-Bio. Avda. Andrés Bello 720, Casilla 447. 3800708 Región de Nuble Chile. Chile. cluengo@ubiobio.cl
}

\begin{abstract}
This research examined the association between occupational fatigue and work absenteeism in 110 female assistant nurses of a high-complexity hospital in Chile. A sociodemographic questionnaire and the Checklist Individual Strength (CIS) scale were used. The results showed that the predominant absenteeism range was 11-29 days. The highest means of fatigue occurred in those with seniority above 2 years, with more than 10 patients in charge and an annual contract. No significant difference was observed between the fatigue means concerning absenteeism, but a significant association was observed between physical fatigue ( $p=0.040, O R=1.054$ ) and service seniority $(p=0.001, O R=1.084)$ with work absenteeism. Finally, we can conclude that physical fatigue and seniority in the clinical service are significant risk factors for the occurrence of absenteeism.
\end{abstract}

Key words Fatigue, Nursing staff, Work absenteeism
Resumen Esta investigación examinó la asociación entre la fatiga y el absentismo en el trabajo en 110 asistentes de enfermería de un hospital altamente complejo en Chile. Se utilizó un cuestionario sociodemográfico y la escala Check Strength Individual Strength. Los resultados mostraron que el intervalo de absentismo predominante fue de 11 a 29 días. Los mayores promedios de fatiga se presentaron en los mayores de 2 años, con más de 10 pacientes en el consultorio y con contrato anual. No hubo diferencias significativas entre la fatiga media en relación con el absentismo, pero hubo una asociación significativa entre la fatiga física ( $p=0,040 ; O R=1,054)$ y la antigüedad en el trabajo ( $p=0,001$; $O R=1,084)$ con el absentismo laboral. Finalmente, se concluye que la fatiga física y la antigüedad en el servicio clínico representaron un factor de riesgo significativo para el absentismo.

Palabra clave Fatiga, Personal de enfermería, Absentismo en el trabajo 


\section{Introducción}

La fatiga laboral, entendida como "una pérdida transitoria de la capacidad para ejecutar un trabajo, consecutiva a la realización prolongada del mismo"', ocupa el tercer lugar en prevalencia como síntoma asociado a la mala salud en las poblaciones de trabajo en general, siendo uno de los mayores factores asociados a morbilidad ${ }^{2}$. Esta posee una relación interdependiente con el trabajo, puesto que se encuentra influida por éste, pero a su vez, también repercute directamente en él. Un aumento en los niveles de fatiga presentado por el personal de salud puede aumentar las posibilidades de cometer errores durante la atención de los pacientes ${ }^{3}$, generando insatisfacción y complicaciones como ulceración, infecciones y caídas ${ }^{4}$, lo que va en desmedro de la seguridad del usuario, comprometiendo la calidad del cuidado, situación especialmente relevante en la atención de enfermería, en la cual se realizan procedimientos de atención directa y muchas veces invasiva. Además, las equivocaciones cometidas por trabajadores fatigados pueden ocasionar efectos negativos con gran alcance en la comunidad, que pueden ir desde errores médicos hasta accidentes automovilísticos ${ }^{5}$. Sumado a esto, la fatiga laboral tiene un impacto negativo en el empleador, ya que los efectos de la fatiga también pueden llevar a un aumento en las tasas de enfermedades ocupacionales y ausentismo laboral ${ }^{2,6}$. Este último, es un tema constante de preocupación en las distintas organizaciones. Una de las áreas de trabajo en donde existen altos índices de ausentismo laboral es en el sector salud, siendo una realidad de importancia en el sector público de salud chileno ${ }^{7}$. El ausentismo puede generar un impacto negativo en la gestión institucional ${ }^{6} \mathrm{y}$ está ligado a grandes costos asociados al reemplazo de personal y al subsidio que dan las entidades de previsión en salud a los trabajadores, es así como en Chile, estos organismos han entregado subvenciones de más de 530 millones de pesos (CLP), sólo en reposición de licencias médicas8. Los altos costos han llevado a las empresas a generar estrategias para disminuir los altos niveles de ausentismo, sin embargo, dichas estrategias no han sido fructíferas.

Cabe destacar, que la mayor cantidad de trabajadores del área de salud es dominada por el estamento auxiliar'. Entre ellos, la mayor concentración se desempeña como auxiliar del área de enfermería, conocidos también como técnicos paramédicos o técnicos en enfermería, quienes son los encargados de la ejecución de actividades básicas pertenecientes al ámbito de enfermería y es en ellos donde se presenta la mayor cantidad de casos de ausentismo ${ }^{10}$. Esta población está principalmente constituida por mujeres ${ }^{11}$, las cuales suelen presentar mayores niveles de ausentismo que los hombres ${ }^{12}$, y quienes además se encuentran sometidas a mayor nivel de carga, ya que responden tanto a las demandas de su trabajo como a demandas familiares y domésticas ${ }^{13}$. No obstante, es una población escasamente estudiada, que ha crecido considerablemente durante los últimos años, presentado un aumento de más del $23 \%{ }^{11,14}$, y está expuesta generalmente a una excesiva carga laboral y a un ritmo de trabajo rápido e intenso ${ }^{15}$. Dichas condiciones propias del área sanitaria pueden terminar por producir un desgaste, tanto en el ámbito físico como en el emocional ${ }^{16}$, lo que puede dejarles más vulnerables ante el estrés, la fatiga y el agotamiento ${ }^{17}$.

Por tanto, considerando lo anteriormente expuesto es que el objetivo de este estudio es determinar la asociación entre la fatiga laboral y el ausentismo en el trabajo en mujeres auxiliares de enfermería de un Hospital de Alta complejidad en Chile. Postulando la hipótesis que: a mayor nivel de fatiga laboral en auxiliares de enfermería mayor es su ausentismo en el trabajo.

\section{Material y método}

Se realizó un estudio analítico de corte transversal, se censo una población de 146 mujeres auxiliares de enfermería (AE), que trabajan en las unidades de Medicina, Cirugía y Neurología pertenecientes a un hospital de alta complejidad en Chile. Obteniendose una población final de 110 (AE), que cumplieron con los criterios de inclusión: a) AE que realicen cuarto turno ; b) que lleve al menos 6 meses trabajando en el servicio; $y$ c) que acepten participar en este estudio firmando consentimiento informado. Fueron considerados criterios de exclusión del estudio, estar con licencia médica, vacaciones y/o permiso legal al momento de recoger los datos, no responder el cuestionario en su totalidad y negarse a participar. Es así como, se excluyeron a $36 \mathrm{AE}$, los motivos fueron: llevar menos de 6 meses trabajando $(16,4 \%)$; negarse a participar del estudio $(2,1 \%)$ y no responder el cuestionario en su totalidad $(3,4 \%)$.

Para el levantamiento de información, efectuada entre los meses de abril-mayo 2018 se utilizaron 2 instrumentos diferentes (auto-administrados, previa lectura de las instrucciones), una 
cédula de datos sociodemográficos y laborales con el propósito de caracterizar la muestra, donde se incorporó dos preguntas para evaluar el ausentismo laboral : ¿Usted se ha ausentado en el trabajo durante los últimos 12 meses? (SI/NO); si la respuesta era afirmativa, se solicitó responder : ¿Cuántos días se ha ausentado en el trabajo en los últimos 12 meses? (Incluye días administrativos, ausencias injustificadas, licencias médicas y accidentes laborales). Como las personas tienden a subestimar sus ausencias ${ }^{12}$, se decidió introducir un párrafo aclaratorio, donde se especificó que aspectos son considerados dentro del ausentismo. Y el Check List Individual Strength o CIS para medir Fatiga Laboral. Este instrumento fue adaptado y validado para Chile en el año 2008 reduciéndose a 15 ítems ${ }^{18}$, de los 20 que originales. A su vez, estos ítems se dividen en dos subescalas las cuales evalúan percepción de fatiga física ( de Cronbach $=0,85)$ y percepción de la fatiga cognitiva ( $\alpha$ de Cronbach $=0.78$ ). Para cuantificar la fatiga se utilizó un promedio de los datos obteniendo un puntaje para la fatiga general. Además, se incluyó un ítem 16 para evaluar la autopercepción de fatiga ${ }^{19}$.

La investigación cumplió con las directrices éticas para la investigación con seres humanos establecidas en la ley decreto 114 que aprueba reglamento de la ley $n^{\circ} 20.120^{20}$, además se solicitó la aprobación del Comité Ético Científico del Hospital en estudio y del director de dicho establecimiento. Todas las participantes leyeron y firmaron dos copias del formulario de consentimiento informado, recalcándoles que su participación es completamente voluntaria, que la información recopilada es de carácter anónimo y confidencial y además que no se verían negativamente afectados por negarse a participar.

El análisis descriptivo de los datos se realizó mediante frecuencias y porcentajes, media y desviación estándar, bajo el supuesto de normalidad medido con Shapiro- Wilk. Con el objetivo de correlacionar las variables del estudio se calculó prueba de ANOVA de un factor, bajo supuesto de normalidad y $\mathrm{H}$ de Kruskal-Wallis cuando los datos no presentaron distribución normal. Para el caso de dos variables cualitativas se aplicó prueba de $\mathrm{ji}^{2}$. Con el objetivo de probar la hipótesis del estudio, se hizo una regresión logística binaria múltiple, con nivel de significación de 0,05. El análisis de los datos fue gestionado con el paquete estadístico SPSS versión 15 en español.

\section{Resultados}

En cuanto a la distribución del ausentismo laboral de los participantes del estudio, un $61 \%$ reporto ausentismo en el trabajo $(\mathrm{N}=67)$ y refirieron ausentarse entre 11 y 29 días.

En relación a fatiga general, el mayor puntaje promedio con una tendencia asociada a presentar mayor fatiga general, lo presentaron las $\mathrm{AE}$ entre 35 a 44 años, con estado civil separadas, que perciben un ingreso económico per cápita entre $\$ 62.090$ y $\$ 106.214$ (CLP), las jefas de hogar, las que tienen al menos 3 hijos y las que indicaron tener 3 o más personas a su cuidado. Dichas observaciones se replican tanto en la fatiga física como en la cognitiva.

Ahora, en relación a la fatiga general según características laborales, las participantes que indicaron tener una experiencia laboral como $\mathrm{AE}$ entre 5 a 16 años, que llevan trabajando de 2 a 5 años en el mismo servicio clínico, que tienen más de 10 pacientes a cargo y que trabajan a contrata, fueron las que presentaron un mayor puntaje promedio con una inclinación a presentar mayor fatiga general y semejante comportamiento sufre la variable fatiga física. En esta última, se visualizó que el promedio más alto se muestra en el personal que trabaja a honorario. En fatiga cognitiva, las participantes que trabajan en medicina, que suman una experiencia laboral total entre 5 a 16 años, que se desempeñan en el mismo servicio clínico por más de 5 años, que tengan más de 10 pacientes a cargo y están contratadas como dotación de planta, presentan un mayor promedio.

En cuanto a la relación entre fatiga laboral y ausentismo en el trabajo (Tabla 1), no hay diferencia significativa entre los promedios del puntaje de fatiga general, física y cognitiva, en relación a los cuatro grupos de ausentismo laboral.

Ahora, con el objetivo de poner a prueba la hipótesis del estudio, se examinaron una serie de ecuaciones de regresión logística binaria de tipo múltiple, entre las cuales se encontró la que se presenta en Tabla 2, donde se observa que fatiga física y años de experiencia en servicio clínico se asociaron significativamente con ausentismo en el trabajo, con $\mathrm{p}=0,040$ y $\mathrm{p}=0,001$, respectivamente.

En relación a fatiga física, se indicó una OR $=1,054$ (IC 95\%: 1,003-1,109) la cual es equivalente a decir que el aumento en un punto de esta dimensión, aumenta en 1,05 veces más el riesgo de ausentarse en el trabajo. Luego, la variable antigüedad laboral en el servicio clínico presento una $\mathrm{OR}=1,084$ (IC 95\%: 1,032-1,139), por lo 
Tabla 1. Asociación entre fatiga laboral y ausentismo en el trabajo de los participantes del estudio

\begin{tabular}{lccccc}
\hline \multirow{2}{*}{ Fatiga laboral } & \multicolumn{4}{c}{ Ausentismo laboral (en días) } & \multirow{2}{*}{ p-valor } \\
\cline { 2 - 5 } & $1-10(\mathrm{~N}=17)$ & $11-29(\mathrm{~N}=24)$ & $30-60(\mathrm{~N}=10)$ & $>60(\mathrm{~N}=16)$ & \\
\hline Fatiga general & $47,53 \pm 14,03$ & $49,25 \pm 15,27$ & $54,20 \pm 10,41$ & $55,06 \pm 19,25$ & $0,447^{\star}$ \\
Fatiga física & $28,88 \pm 8,56$ & $30,17 \pm 9,73$ & $30,20 \pm 7,36$ & $32,31 \pm 11,79$ & $0,788^{*}$ \\
Fatiga cognitiva & $18,65 \pm 7,97$ & $19,08 \pm 8,30$ & $24,00 \pm 8,29$ & $22,75 \pm 8,96$ & $0,226 \dagger$ \\
\hline * Prueba de ANOVA + Prueba H de Kruskal-Wallis.
\end{tabular}

Tabla 2. Regresión logística binaria entre ausentismo laboral con respecto a fatiga física, fatiga cognitiva y años de experiencia en el servicio clínico.

\begin{tabular}{lccccccc}
\hline \multicolumn{1}{c}{ Variables independientes } & Coef B & $\begin{array}{c}\text { Error } \\
\text { típico }\end{array}$ & Wald & p-valor & OR & \multicolumn{2}{c}{ IC 95\% para OR } \\
\hline Fatiga física & 0,053 & 0,026 & 4,234 & 0,040 & 1,054 & 1,003 & 1,109 \\
Fatiga cognitiva & $-0,017$ & 0,028 & 0,380 & 0,537 & 0,983 & 0,931 & 1,038 \\
$\begin{array}{l}\text { Antigüedad laboral en el servicio } \\
\text { clínico }\end{array}$ & 0,081 & 0,025 & 10,183 & 0,001 & 1,084 & 1,032 & 1,139 \\
Constante & $-1,508$ & 0,782 & 3,721 & 0,054 & 0,221 & & \\
\hline
\end{tabular}

que trabajar un año más en el mismo servicio clínico, incrementa en 1,084 veces más el riesgo de ausentarse en el trabajo.

\section{Discusión}

Este estudio da cuenta, de la asociación entre fatiga laboral y ausentismo en el trabajo en mujeres Auxiliares de Enfermería.

Referente al Ausentismo laboral, se observó que gran parte de la población refirió ausentarse entre 11 y 29 días, lo que concuerda con lo expuesto por Mesa y Kaempffer ${ }^{21}$ en donde la cantidad de días promedio de ausentismo en el sector hospitalario fue de 14,3 días. Luego, al analizar la fatiga general se encontró que el tramo de edad de 35 a 44 años tuvo el promedio más alto de fatiga, tanto general, física como cognitiva. Se podría pensar que el grupo etario más longevo podría tender a manifestar mayor índice de fatiga, situación que no se presentó. Esto puede estar dado porque las personas de edad más avanzada presentan generalmente menos sobrecarga de trabajo ya que, existe mayor colaboración de sus pares para realizar tareas que requieren de más esfuerzo físico. En lo que respecta al estado civil de las participantes podemos apreciar que las separadas tienen un promedio más alto de fatiga general y física. Al respecto Portero y Vaquero ${ }^{22}$ mencionan que el matrimonio no tiene gran influencia en el desgaste profesional, sino que la incidencia estaría marcada por el apoyo que se recibe por parte de la pareja. En cuanto al ingreso económico per cápita, quienes reportan los niveles más bajos de fatiga se encuentran en los grupos que declararon menores ingresos. Luego, tener menor ingreso llevaría a aumentar la preocupación de dar sustento a la familia cubriendo todas las necesidades en base a un presupuesto familiar bajo. Estos resultados se refuerzan con lo encontrado por Ansoleaga et al. ${ }^{23}$ quienes determinaron que el distrés (estrés negativo) se asoció con estrechez económica. Por otro lado, las participantes que se consideraron jefas de hogar tuvieron un mayor promedio en todos los tipos de fatiga, a diferencia de las que no lo eran. Esto podría deberse a que ser jefa de hogar involucra lidiar con variadas responsabilidades y participar en la resolución de problemas, tanto en el hogar como en el trabajo. Muchas de las participantes que son jefas, no tan solo se deben encargarse del cuidado del hogar sino también del cuidado del esposo y de los hijos, sumado a las responsabilidades laborales, lo que podría exacerbar los conflictos de roles ${ }^{13}$. Según Díaz y Mauro ${ }^{24}$ una gran proporción de trabajadoras del área sanitaria reporta una muy alta carga de trabajo, sobrepasado las 60 horas semanales de trabajo doméstico y de trabajo remunerado, lo que podría equivaler a realizar más de 12 horas a la semana solo de trabajo no remunerado. En relación a al número de hijos, quienes tenían más hijos tendían a presentar mayores niveles de fatiga en comparación 
con aquellos que no tenían hijos. Esta situación se replica en el número de personas a su cuidado, en donde las participantes que asumen el rol de cuidador de más personas tienden a tener niveles más altos de fatiga que aquellos que no tienen personas a su cuidado. Se puede apreciar una tendencia directamente proporcional entre el número de cargas y el promedio de fatiga. Seguel ${ }^{19}$ afirma que la presencia de hijos implica mayor fatiga para el trabajador de enfermería, de hecho mientras mayor sea la cantidad de hijos que tiene el trabajador de enfermería, mayor será la fatiga, ya sea general, física y cognitiva. Esto concuerda con lo encontrado por Jones et al. ${ }^{3}$, quienes expusieron que la presencia de hijos era uno de los factores que estaba significativamente asociado con mayores probabilidades de manifestar un estado cansancio. Estos resultados pueden estar relacionados a que atender las demandas familiares y domésticas, que incluyen el cuidado de los hijos y/o algún pariente puede generar mayor desgaste cuando a éstas se suman las demandas laborales.

En este mismo sentido, el mayor promedio de fatiga general y en las subescalas física y cognitiva se presentó en aquellas participantes que llevaban entre 5 y 16 años trabajando como AE. Al respecto un estudio cubano encontró que el tiempo de trabajo total tenía asociación con la fatiga y el estrés ${ }^{25}$. Por otro lado, las participantes que tienen mayor número de pacientes a cargo presentan una tendencia a tener promedios más altos de fatiga. Al tener menos pacientes, menores serán las tareas que se deben realizar y por ende el esfuerzo físico también estará disminuido.

En lo que refiere a la relación entre fatiga laboral y ausentismo en el trabajo, a pesar de que existe una tendencia al aumento de los promedios tanto para la escala de fatiga general como para la subescala de fatiga física a medida que aumentan los días de ausencia, la asociación no se considera significativa. En cuanto a la subescala de fatiga cognitiva, no existe una asociación entre el promedio y los días de ausentismo. Según Roelen et al. ${ }^{26}$ en su estudio en trabajadores de cuello blanco encontró que la fatiga prolongada se asocia prospectivamente con ausencias a largo plazo, lo que produce interrupción en el adecuado funcionamiento laboral. En otros estudios del mismo autor $^{2}$ en empleados de oficina se encontró que la fatiga se asoció de manera prospectiva, tanto con altos días de ausencia por enfermedad como con el número de episodios de ausencia durante el seguimiento de 1 año. Estos estudios coinciden con lo encontrado por Bultmann et al. ${ }^{27}$ en una investigación realizada en trabajadores daneses, en donde se observó que el aumento de 1 desviación típica en la fatiga predijo un aumento del $16 \%$ en el riesgo de ausentarse por enfermedad, sin embargo en otros análisis realizados en este mismo estudio, se encontró que la fatiga es un factor fuerte e independiente para predecir la ausencia por enfermedad en los hombres, pero no así en las mujeres. En dicho estudio no quedo clarificado por qué la relación entre la fatiga y el ausentismo se presentaba con mayor fuerza en los hombres que entre las mujeres, aunque se sugerían algunas teorías como que los motivos de fatiga sean diferentes para cada sexo. Además, se agregaba el hecho de que los hombres desempeñaban tipos de trabajo incompatibles con el aumento en los niveles de fatiga, tales como aquellos que requerían de mayor esfuerzo físico, por lo que cuando se sentían fatigados no podían cumplir adecuadamente sus labores, lo que los llevaba a ausentar. La misma situación no se daba en las mujeres ya que éstas tienen un mayor éxito cuando se refiere a lidiar con la fatiga lo que repercute en tener una mejor adaptación en su capacidad de trabajo. Resultados como estos nos llevan a pensar, que si bien la fatiga si presenta una influencia en el ausentismo, cuando nos referimos a género femenino, ellas presentan otros motivos que las llevan a no asistir a su lugar de trabajo.

Respecto a la asociación entre la jefatura de hogar y el ausentismo en el trabajo no se encontró significativa. De esto se deduce que la jefatura es un factor independiente que no conduce a ausentarse. Sin embargo, ésta indirectamente puede llevar a aumentar los días de ausencia, ya que las labores y responsabilidades que implica ser jefa de hogar pueden derivar en otros factores tales como, agotamiento, estrés entre otras. La multiplicidad de roles que se necesitan llevar a cabo para cumplir tanto las demandas del hogar, como las laborales pueden producir excesiva carga de trabajo $^{19}$ y ser esta ultima la desencadenante del ausentismo.

Al analizar la regresión logística binaria de tipo múltiple, de todas las diferentes pruebas que se realizaron se evidenció que sólo la fatiga física y la antigüedad laboral en el servicio clínico se asociaron significativamente con ausentismo en el trabajo. En relación a fatiga física, se encontró que, si hay un aumento de un punto en esta dimensión, aumenta en 1,05 veces más el riesgo de ausentarse en el trabajo, relación que no se evidencio en la fatiga cognitiva. En tanto la hipótesis planteada que "a mayor nivel de fatiga laboral en auxiliares de enfermería mayor es su ausentismo en el trabajo", se acepta parcialmente. 
Otro hallazgo relevante fue que trabajar un año más en el mismo servicio clínico, incrementa el riesgo de ausentarse en el trabajo 1,084 veces más, de lo que se puede deducir que es un factor de riesgo que puede tener aún más influencia en el ausentismo laboral que la misma fatiga física. En este sentido, un estudio noruego encontró que la fatiga física inicial se asociaba significativamente con alta ausencia por enfermedad al año de seguimiento en la atención psiquiátrica, el hogar de ancianos y la atención domiciliaria, sin embargo, esta asociación no se reflejó en la fatiga mental ${ }^{28}$, resultados que concuerdan con la presente investigación. Además, en el mismo estudio se encontró que las asociaciones entre la fatiga y la ausencia por enfermedad se fortalecie- ron cuando había un aumento de las demandas laborales y conflicto de roles. Asimismo, Vera et al. ${ }^{18}$, quienes estudiaron la fatiga en trabajadores mineros, realizaron un modelo de regresión lineal múltiple para fatiga física y cognitiva ajustado a la edad. De él se desprendió que existe una asociación significativa $(\mathrm{p}=0,008)$ entre la fatiga física y la capacidad predictiva positiva de los años trabajando en el cargo.

Finalmente, se concluye que la fatiga física y antigüedad laboral en el servicio clínico representaron un factor de riesgo significativo para presentar ausentismo laboral. Luego, es importante seguir realizando estudios en la temática y en esta unidad de análisis como integrante fundamental del equipo de enfermería.

\section{Colaboradores}

CL Martínez participó en: concepción y diseño del trabajo, análisis e interpretación de datos, redacción del manuscrito, revisión crítica del manuscrito, otorgó asesoría estadística y administrativa e aprobó su versión final. Los autores SP Moraga, CS Paredes, AS Vásquez, CM Villarroel, participaron indistintamente en la concepción y diseño del trabajo, recolección y obtención de los resultados, análisis e interpretación de datos, redacción del manuscrito, revisión crítica del manuscrito y aprobación de su versión final. 


\section{Referencias}

1. Useche L. Fatiga laboral. Avances en Enfermería 1992; 10:89-103.

2. Roelen C, Bültmann U, Groothoff J, Twisk J, Heymans M. Risk reclassification analysis investigating the added value of fatigue to sickness absence predictions. Int Arch Occup Environ Health 2015; 88(8):1069-1075.

3. Jones G, Hocine M, Salomon J, Dab W, Temime L. Demographic and occupational predictors of stress and fatigue in French intensive-care registered nurses and nurses' aides: A cross-sectional study. Int J Nurs Stud 2015; 52(1):250-259.

4. Kalisch B, Tschannen D, Lee H, Friese C. Hospital Variation in Missed Nursing Care. Am J Med Qual 2011; 20(10):1-9.

5. Caruso C. Reducing Risks to Women Linked to Shift Work, Long Work Hours, and Related Workplace Sleep and Fatigue Issues. J Women's Health 2015; 24(10):789-794.

6. Silva F, Felli V, Martinez M, Mininel V, Pelegrini A. Association between work ability and fatigue in Brazilian nursing workers. Work 2016; 53(1):225-232.

7. La Tercera.com [Sede Web]. Ausentismo Laboral en el sector salud duplica promedio nacional; 2016. [acceso 2017 Nov 20]. Disponible en: http://www.latercera. com/noticia/ausentismo-laboral-en-sector-de-salud-duplica-promedio-nacional/

8. Soto A, Herrera R, Fuentes R. Efecto de la seguridad social en la duración del ausentismo laboral en el Servicio Salud de Ñuble: Un análisis de supervivencia. Rev Med Chil 2015; 143(8):987-994.

9. Chile. Superintendencia de salud. Estadísticas de Prestadores Individuales de Salud, Período: 1 de abril de 2009 al 30 de junio de 2017. Santiago: Unidad de Registro, Intendencia de Prestadores de Salud, Superintendencia de Salud; 2017.

10. Rojas L. Estudio descriptivo del ausentismo laboral en trabajadores del sistema público de salud en Chile [tesis]. Santiago: Universidad de Chile; 2014.

11. Chile. Ministerio de Salud (MS). Subsecretaría de Redes Asistenciales División de Gestión y Desarrollo de las Personas. Departamento de Planificación y Control de Gestión de RHS. Informe sobre brechas de personal de salud por servicio de salud, Glosa 01, letra i. Ley de Presupuestos Año 2017. Santiago: MS; 2017.

12. Mendoza R. Insatisfacción laboral como predictor del ausentismo en un hospital público. Rev Med Chil 2015; 143(8):1028-1033.

13. Uzoigwe A, Low W, Noor S. Predictors of Work-Family Role Conflict and Its Impact on Professional Women in Medicine, Engineering, and Information Technology in Nigeria. Asia Pac J Public Health 2016; 28(7):629-637.

14. Chile. Subsecretaría de Redes Asistenciales. División de Gestión y Desarrollo de las Personas, Dpto. Planificación de RHS y Control de Gestión. $1^{\circ}$ informe sobre los prestadores individuales de salud inscritos en el registro nacional de la superintendencia de salud (SIS) y la dotación de personal del sector público. Santiago: Subsecretaría de Redes Asistenciales; 2014.

15. Padilla C, Palmeiro Y. Effort-Reward Imbalance and Burnout Among ICU Nursing Staff: A Cross-Sectional Study. Nursing Res 2017; 66(5):410-416.
16. Darli R, Silva L, Mendes A, Robazzi M. Carga horaria de trabajo de los enfermeros y su relación con las reacciones fisiológicas de estrés. Rev. Latino-Am. Enfermagem 2014; 22(6):959-65.

17. Lima J, Silva R, Santos F, Souza D, Bittencourt F, Reis L. Psychosocial factors and prevalence of burnout syndrome among nursing workers in intensive care units. Rev Bras Ter Intensiva. 2015; 27(2):125-133.

18. Vera A, Carrasco C, Vanegas J, Contreras G. Fatiga Física y Fatiga Cognitiva en Trabajadores de la Minería que Laboran en Condiciones de Altitud Geográfica. Relación con el Mal Agudo de Montaña. Cienc Trab 2008: 10(29):90-94.

19. Seguel F. Factores relacionados con fatiga y burnout en personal de enfermería en relación a la presencia de exceso de trabajo [tesis]. Concepción: Universidad de Concepción; 2014.

20. Chile. Ley 20.120 del 22 de septiembre de 2006. Sobre la investigación científica en el ser humano, su genoma, y prohíbe la clonación humana del personal estatutario de los servicios de salud. Biblioteca del Congreso Nacional de Chile 2006; 22 sep.

21. Mesa F, Kaempffer M. 30 años de estudio sobre ausentismo laboral en Chile: una perspectiva por tipos de empresas. Rev Med Chil 2004; 132(9):1100-1108.

22. Portero S, Vaquero M. Desgaste profesional, estrés y satisfacción laboral del personal de enfermería en un hospital universitario. Rev. Latino-Am. Enfermagem 2015; 23(3):543-552.

23. Ansoleaga E, Díaz X, Mauro A. Asociaciones entre distrés, riesgos psicosociales y calidad del empleo en trabajadores/as asalariados/as en Chile: una perspectiva de género. Cad Saude Publica 2016; 32(7):e00176814.

24. Diaz X, Mauro A. Riesgos psicosociales y salud mental de trabajadores y trabajadoras del sector salud en Chile. Santiago: Centro de Estudios de la Mujer; 2012.

25. Almirall P, Del Castillo N, Parada C, Reyes L, Hernandez J, González A. Estrés y fatiga en el ámbito hospitalario: un estudio con enfoque de género. Revista Cubana de Salud y Trabajo 2013; 13(1):46-50.

26. Roelen, C, Van Rhenen W, Groothoff J, Van der Klink J, Bültmann U. Prolonged fatigue is associated with sickness absence in men but not in women: prospective study with 1-year follow-up of white-collar employees. Int Arch Occup Environ Health 2013; 87(3):257263.

27. Bültmann U, Nielsen M, Madsen I, Burr H, Rugulies R. Sleep disturbances and fatigue: independent predictors of sickness absence? A prospective study among 6538 employees. Eur J Public Health 2012; 23(1):123-128.

28. Roelen C, Bültmann U, Groothoff J, Van Rhenen W, Magerøy N, Moen B, Pallesen S, Bjorvatn B. Physical and Mental Fatigue as Predictors of Sickness Absence Among Norwegian Nurses. Res Nurs Health 2013; 36(5):453-465.

Artigo apresentado em 03/04/2019

Aprovado em 20/08/2019

Versão final apresentada em 03/10/2019 
